\title{
PRECISE COMPACT SYSTEM FOR IONIZING RADIATION DETECTION AND SIGNAL PROCESSING WITH ADVANCED COMPONENTS INTEGRATION AND ELECTRONIC CONTROL
}

\author{
Jakub Navařík - Petr Novák - Jiří Pechoušek \\ Libor Machala - Dalibor Jančík - Miroslav Mašláň *
}

\begin{abstract}
Quality and performance of a detection system are the crucial parameters in all nuclear physics experiments. This system serves as a source of all signals and noises to be processed. Better performance, higher amplification and lower noises occurrence simplify subsequent signal analysis. In the field of Mössbauer spectroscopy, the spectrum quality and Mössbauer effect are crucial parameters which are affected especially by the quality of the detection system. These parameters were evaluated for different types of a detection setup. Finally an improvement of the spectrum quality by $15 \%$ and Mössbauer effect by $7 \%$ has been achieved for the natural iron reference absorber measurement in comparison with previous version of the detection system.
\end{abstract}

K e y w o r d s: gamma ray detection, signal processing, Mössbauer spectroscopy, Mössbauer spectrum

\section{INTRODUCTION}

Almost every nuclear physics experiment is based on a detection of a specific type of radiation followed by a processing of the detector output signal. For those purposes, various types of detection systems can be utilized, usually consisting of several separated devices with more or less complicated setup.

Advanced detection systems containing ionizing radiation detectors and signal processing electronics have been described in many papers. The end electronics system unit for processing of the signal of the semi conductive ionizing radiation detectors is described in [1]. Developed integrated circuit processes signal and sends data to a master system. In this case, the ionizing radiation detector is used as a separated unit. In [2] the system for the fast pulses of the ionizing radiation detectors digitization is described, where output data are transferred to a control system. In this case, the detector is used as a separated unit as well. The integrated circuit for signal processing, the development of which is described in [3], where the system contains parts for analog signal processing, however, without a detector. In [4] a technique for optimization of a single channel analyzer is described. A development of a fast scintillation detector is described in [5]. This detector contains integrated, programmable high voltage supply. In [6] a portable spectrometric system for gamma radiation detection is proposed. The system is based on PIN diode detector and contains signal processing electronics.

Only partial solutions of detection systems have been presented in available literature. There is no single, stand- alone device with all the necessary devices integrated (detector, pre-amplifier, amplifier, discriminator and singlechannel analyzer), especially with the dimension below $30 \mathrm{~cm}$.

Detection systems for nuclear physics experiments, including detectors and electronics integrated in a single body or a single optimized chain have also been described in the selected patents. In [7] a design of a system with Geiger-Müller detector and control electronics is described. In [8] a detection system including semiconductor diodes, a charge amplifier and a discriminator as a complete detection system has been proposed. The signal is being processed by using a microcontroller there. This system is very complex, but all the devices are separated. A detection system with ionizing chamber is described in [9]. It contains electronics for signal processing as well, including a microcontroller, a power supply, a battery, and analog amplifiers. This device contains even communication interface WiFi, GPS, RS-232 and USB. A complete detection system with signal processing based on a scintillation detector and expansion blocks is described in [10]. In [11] a Cadmium-Zinc-Tellurium detector with signal processing electronics is described. It contains a detector, signal amplifiers, and filters. There is no pulse registration unit and a counter unit has to be connected externally. In [12] a design of a detection system for continuous monitoring and ionizing radiation detection, especially for dosimetry measurements is described.

In the summary an ionizing radiation detector is represented by a main block mostly without integrated high voltage power supply, or without a signal processing unit integrated, or there are no pre-amplifiers and amplifiers in

* Regional Centre of Advanced Technologies and Materials, Department of Experimental Physics, Faculty of Science, Palacky University, 17. Listopadu 12, 77146 Olomouc, Czech Republic, jakub.navarik@upol.cz, jiri.pechousek@upol.cz 


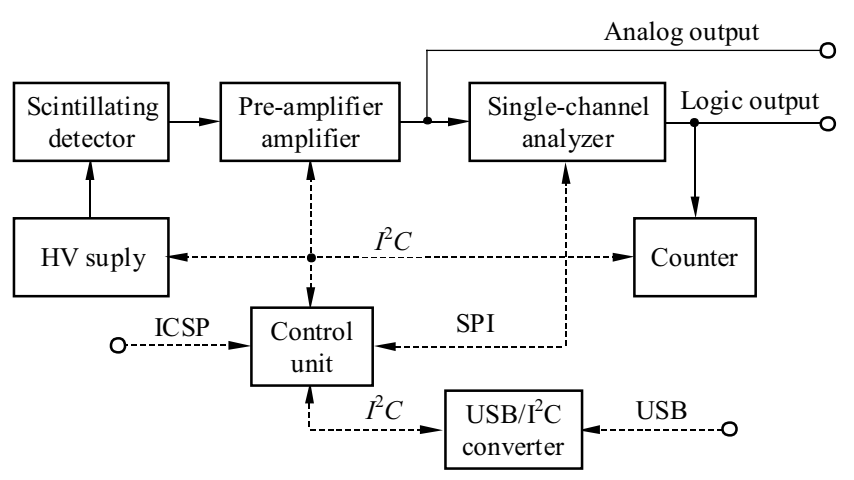

Fig. 1. Block diagram of IND detection system

some solutions. Each additional electronic block is usually realized as a separated device which has to be connected with the detection system externally.

The detection system described in this paper is designed to be complex, small and powerful. We consider it as a continuation of previously published papers and documents $[4,5,13-17]$. It represents a single device which consists of a scintillating detector, an electronically controllable high voltage power supply, a pre-amplifier, an electronically controllable amplifier, an electronically controllable single channel analyzer, a counter (which provides multi-channel analysis) and a microcontroller. Such system has been primarily designed for usage in the field of Mössbauer spectroscopy; however it can be applied in other fields as well.

\section{IND DETECTION SYSTEM DESCRIPTION}

The main purpose of this system is to perform gammaray spectroscopy (especially applied in Mössbauer spectroscopy).

All parts of the detection system working in close-packed configuration are handled by a control unit through various internal communication interfaces. Thanks to that, a high voltage supply, amplification and a single channel analyzer (SCA) are simply externally electronically controllable using a standard USB interface. Some parts are moreover electronically re-programmable via In-Circuit Serial Programming interface (ICSP). This system was named Intelligent Nuclear Detector (IND).

As an up-to-date, IND detection system has been designed to be powerful, small and easy to use. Following that idea, it has integrated all the necessary parts to operate, including a scintillating detector, a pre-amplifier, an amplifier, SCA, a counter and a control unit that are connected together as it is shown in Fig. 1.

According to the previously published experimental results [13], as the best suitable scintillating material YAP:Ce (Yttrium Aluminum Perovskite, doped with Cerium; diameter $28 \mathrm{~mm}$, thickness $0.4 \mathrm{~mm}, 30 \mathrm{~nm}$ aluminum film) with photomultiplier the R6095 tube (HAMAMATSU) has been chosen. This provides negative voltage output pulses.
Two stage amplification of the registered signal is provided by the inverting pre-amplifier (fixed amplification $80 \times$ ) and the fine amplifier (up to $81 \times$ adjustable amplification). Both amplifiers are based on the AD8038 operational amplifier (Analog Devices). Then, polarity of output pulses is positive and this signal is driven to ana$\log$ output of IND and/or to SCA.

Amplification and high voltage level are electronically controllable. For this purpose, the AD5252 (Analog Devices) digital potentiometer is used, which is set via standard $\mathrm{I}^{2} \mathrm{C}$ interface by the control unit. As it is shown in Fig. 2, the AD5252 is a dual device. In presented system, the first digital potentiometer is used for a control of amplification (Fig. 2 left), and the second one for a control of the high voltage level (Fig. 2 right). The C9028-01 (HAMAMATSU) high voltage supply is used. The high voltage level has a range from 0 to $1250 \mathrm{~V}$ and it is set by voltage level on the adjustment input of the C9028-01 ( $0-5 \mathrm{~V}$ means $0 \%-100 \%)$. The digital potentiometer is set by a control unit, using standard $\mathrm{I}^{2} \mathrm{C}$ interface.

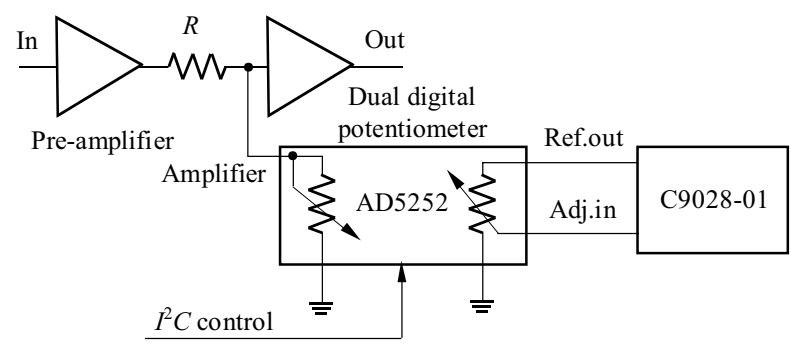

Fig. 2. Amplification (left) and high voltage level control (right)

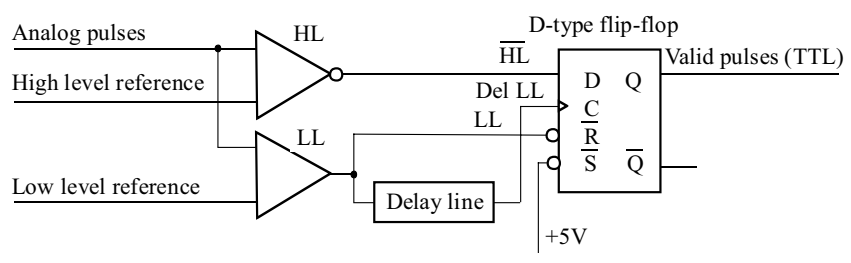

Fig. 3. SCA simplified schematic, realization with two comparators [4]

SCA [4] is based on the discriminator unit with two fast ADCMP600 (Analog Devices) comparators with 3.5 ns response time, D-type flip-flop trigger and DS1023100 (Maxim Integrated) programmable delay line. The simplified schematic of SCA is illustrated in Fig. 3.

The analog signal from the detector output is driven to the both of comparators. One comparator represents HL (higher reference voltage level), the other one LL (lower reference voltage level) of the discriminator window. As it is shown in Fig. 3, a signal from HL is inverted and then driven directly to data input (D), while LL is driven directly to the inverted reset input $(\overline{\mathrm{R}})$ and after delaying as delLL to the clock input (C) of the D-type flip-flop trigger. There is a function time diagram in Fig. 4. 
Three different cases of pulse analyzes (registration) are shown in Fig. 4. including a pulse with the amplitude lower than LL (I, recognized as non-valid), the pulse with the amplitude directly between LL and HL (II, recognized as valid), and the pulse with the amplitude higher than HL (III, recognized as non-valid).

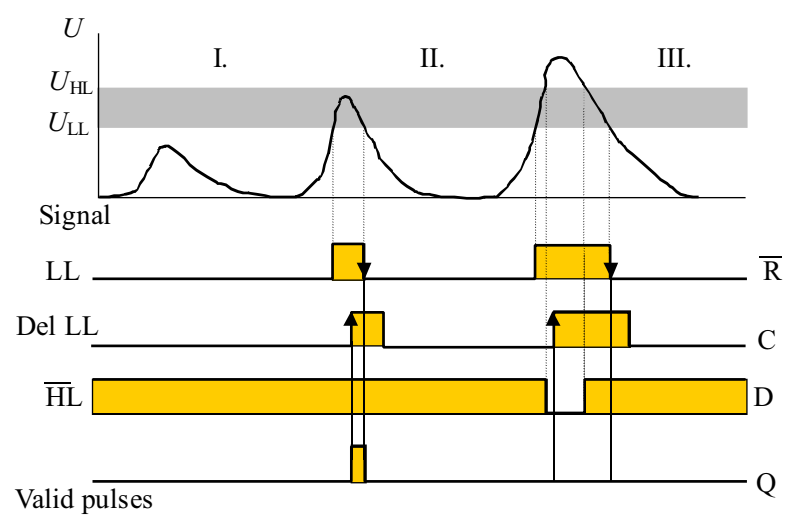

Fig. 4. Function time diagram of SCA [4]

In the first case (I), the flip-flop trigger is being hold in reset state by logic 0 on $\overline{\mathrm{R}}$ input. In the second case (II) the situation is very different. Exceeding of LL value generates logic 1 on $\overline{\mathrm{R}}$ input and the flip-flop trigger is active. After a proper delay (optimization of delay is explained in Section 3.3), there is a C signal generated and because of not-exceeding HL there is a logic 1 on $\mathrm{D}$ input. This generates the logic 1 on $\mathrm{Q}$ output of the flipflop trigger (it lasts after $\overline{\mathrm{R}}$ input is returned to a logic 0 ). Finally in the third case (III), the situation is similar to the second one. There is a logic 1 on $\overline{\mathrm{R}}$ input (the flip-flop trigger is active), after a proper delay, C signal is generated as well, however, because of exceeding of HL, there is a logic 0 on D input of the flip-flop trigger and thus no signal is generated on output Q. This behavior provides reliable selecting of the valid pulses only.

Additionally in a combination with the 74HCT4020 14-bit counter and the PIC16F819 programmable microcontroller (Microchip), this SCA is used to perform a multi-channel analysis (MCA). This MCA is provided by scanning a complete range of SCA step by step. In every step, the number of detected pulses is counted by a counter and then being read out and stored by the microcontroller which is electronically reprogrammable via standard ICSP interface.

The IND function is programmed and controlled by the main control unit, which is based on the PIC16F819 programmable microcontroller. This microcontroller is operating on $8 \mathrm{MHz}$ frequency and its purpose is to communicate with the other parts of the IND system and to store all the operating parameters. For this communication, various standard busses are used. $\mathrm{I}^{2} \mathrm{C}$ interface provides communication with the AD5252 digital potentiometer and a counter unit (setting up of amplification, high voltage level and reading out counter unit's memory). Standard SPI interface is used to set up the MCP4922 (Microchip) digital-to-analog converter (for generating of reference voltage levels for SCA). Because of the possibility to upgrade a program of the control unit, the ICSP interface is led out through IND power \& communication cable. For the communication with PC, an $\mathrm{I}^{2} \mathrm{C} / \mathrm{USB}$ interface converter is used and so the IND can be connected and controlled easily by any ordinary $\mathrm{PC}$ via standard USB interface.

For the precise adjustment of all the controllable parameters a control PC application developed in LabVIEW $^{\mathrm{TM}}$ is used.

\section{MEASUREMENT}

The main field of interest for this detection system is Mössbauer spectroscopy, especially on ${ }^{57} \mathrm{Fe}$ nuclei. In that case ${ }^{57} \mathrm{Co}$ source with emitted gamma ray energy of $14.4 \mathrm{keV}$ is used hence all parameters are optimized for the detection of this energy; however it can be simply optimized for different energies (optimization is described in the following sections).

\subsection{High Voltage and Amplification optimization}

The internal SCA is capable to analyze pulses in the amplitude range from 0 to $3.3 \mathrm{~V}$. It means, that all the operating parameters must be optimized to keep $14.4 \mathrm{keV}$ photo-peak between this voltage levels, with as high resolution as possible at the same time. For this purpose the high voltage level and the amplification has to be optimized. The virtual instrument based on National Instruments HW and SW was used as a standard multi-channel analyzer for comparison [18], with the fast pulse processing algorithm [19].

The C9028-01 high voltage power supply is capable to provide $1250 \mathrm{~V}$ as maximum (which is approximately $80 \%$ of the maximum allowed load of the R6095 photomultiplier tube). Thus the high voltage level was set to maximum to obtain the gain $\approx 10^{7}$, and the amplification was adjusted to obtain a clear $14.4 \mathrm{keV}$ photo-peak in measured MCA.

In Fig. 5, there are MCAs of the output signal of the amplifier. The first MCA (I) represents all energies, the second MCA (II) has suppressed low energies (using Aluminum filter) and thus $14.4 \mathrm{keV}$ photo-peak is determined. The measurement was done with maximal high voltage level and amplification; for the time interval of $100 \mathrm{~s}$, using NI PCI-5124 digitizer (National Instruments) [17].

\subsection{Amplifier output signal parameters}

Formerly, the most used scintillating crystal in MS was NaI:Tl, however, it is not very well suitable for gamma photon detection with high-activity sources. Output pulses provided with NaI:Tl crystal are about eight 


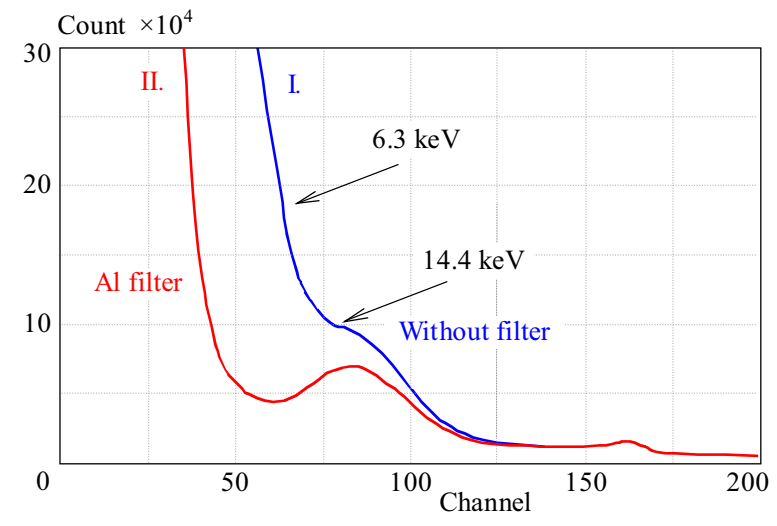

Fig. 5. YAP:Ce MCAs measured using NI PCI-5124

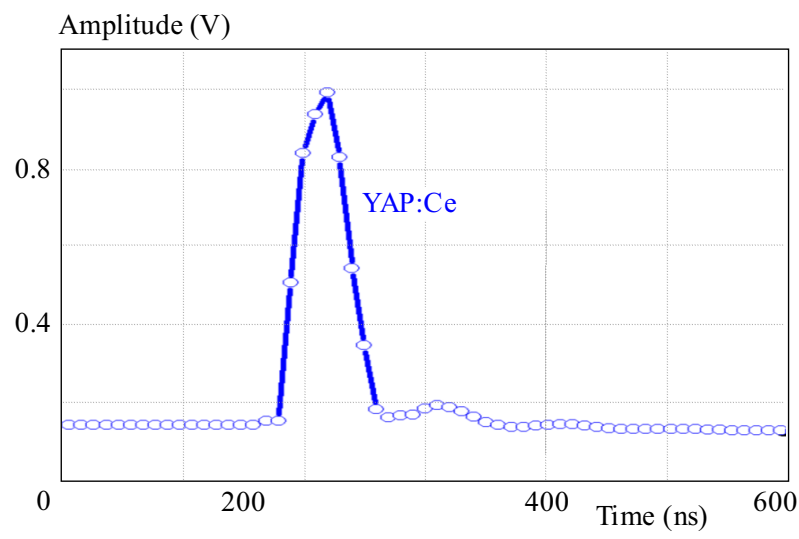

Fig. 6. YAP:Ce average pulse recorded using NI USB-5133

Table 1. Mössbauer effect and spectrum quality dependence on selected delay

\begin{tabular}{cccccccccccccc}
\hline Delay $(\mathrm{ns})$ & 20 & 25 & 30 & 35 & 40 & 45 & 50 & $\mathbf{5 5}$ & 60 & 65 & 70 & 75 & 80 \\
\hline Effect (\%) & 10.32 & 13.58 & 14.51 & 15.25 & 15.47 & 15.23 & 15.69 & $\mathbf{1 5 . 8 4}$ & 13.80 & 14.09 & 13.69 & 13.09 & 11.03 \\
Quality & 533 & 922 & 1053 & 1163 & 1197 & 1159 & 1231 & $\mathbf{1 2 5 4}$ & 952 & 992 & 937 & 857 & 608 \\
\hline
\end{tabular}

Table 2. MS'96 SCA and IND internal SCA - comparison of the spectrum quality and Mössbauer effect

\begin{tabular}{lcc}
\hline & Spectrum quality & Mössbauer effect (\%) \\
\hline MS'96 SCA & 1092 & 14.78 \\
IND internal SCA & 1254 & 15.84 \\
\hline
\end{tabular}

times longer than in the case of YAP:Ce crystal. For this reason, YAP:Ce has been selected as the best suitable scintillating crystal.

After optimizing the high voltage and the amplification, the average pulse was recorded. The pulses with $1 \mathrm{~V}$ amplitude was selected $( \pm 5 \%)$ and then 100000 pulses were collected and averaged. The shape of the average pulse was recorded using NI USB-5133 digitizer (National Instruments) with $100 \mathrm{MS} / \mathrm{s}$ real-time sampling rate.

As can be seen in Fig. 6, both rise and fall time was measured as $50 \mathrm{~ns}$. The complete pulse length is only $100 \mathrm{~ns}$. It means, that YAP:Ce is not so vulnerable to pile-up effect [19] and it is better suitable for high-activity sources.

\subsection{SCA optimization with Mössbauer spectra measurements}

The final optimization step was based on the adjustment of the internal SCA. This analyzer is based on electronically controllable delay line; therefore this delay line must be optimized properly, according to signal parameters. The DS1023-100 delay line has a variable delay from $0 \mathrm{~ns}$ to $255 \mathrm{~ns}$, with $1 \mathrm{~ns}$ step. The delay is set as 8-bit number, which represents from $20 \mathrm{~ns}$ up to $275 \mathrm{~ns}$ time delay.

As it is shown in Fig. 7, a different time delay has a significant effect on the MCA shape. There are three
MCAs registered with different time delays. As the first (I), there is the MCA for 20 ns time delay. In this case, the $14.4 \mathrm{keV}$ photo-peak is the widest. As the second (II), there is the MCA for $50 \mathrm{~ns}$ delay. In this case, the $14.4 \mathrm{keV}$ photo-peak is not so wide, on the other hand, it is better visible (distinguishable) in comparison with the first case. As the last (III), there is the MCA for $80 \mathrm{~ns}$ time delay. It is obvious that the $14.4 \mathrm{keV}$ photo-peak is the least distinguishable and its width is the lowest as well. Thus it can be supposed that the best parameters are a compromise between the $14.4 \mathrm{keV}$ photo-peak width and distinguishability.

For a precise optimization, MCAs was measured for time delays from $20 \mathrm{~ns}$ to $80 \mathrm{~ns}$, with $5 \mathrm{~ns}$ step. The position of $14.4 \mathrm{keV}$ photo-peak was determined for each MCA and then transmission Mössbauer spectrum was measured using MS'96 Mössbauer spectrometer [14,15] (each spectrum was measured for one hour, using the natural iron reference absorber). All measured spectra were normalized and compared (Mössbauer effect and spectrum quality).

The statistical quality of measured Mössbauer spectra is determined by the expression

$$
Q=\frac{\varepsilon^{2}}{\varepsilon+2} N_{\infty}
$$

where $\varepsilon$ is the resonant effect

$$
\varepsilon=\frac{\left|N_{\infty}-N_{0}\right|}{N_{\infty}}
$$

where $N_{\infty}$ represents the number of the counts of the background and $N_{0}$ represents the number of the counts at the minimum resonance peak. These equations were used to obtain values presented in Tab. 1. 


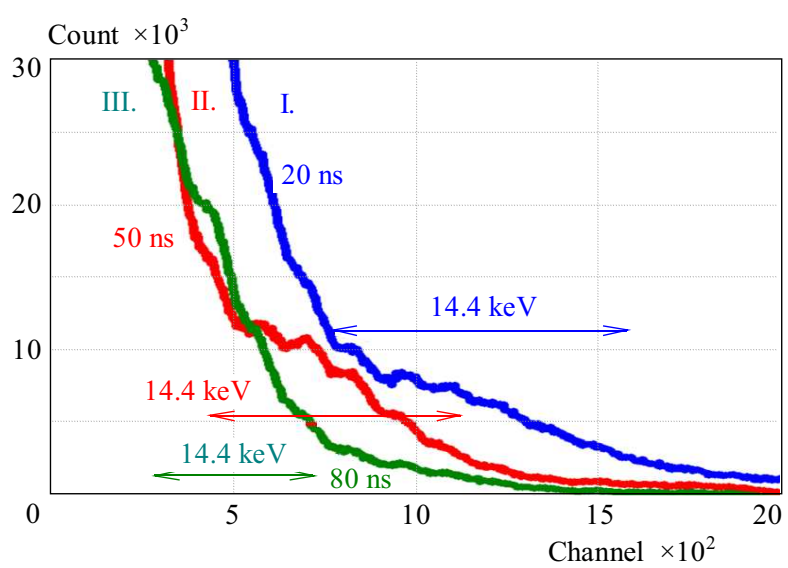

Fig. 7. YAP:Ce MCA shape dependence on selected delay, IND internal SCA

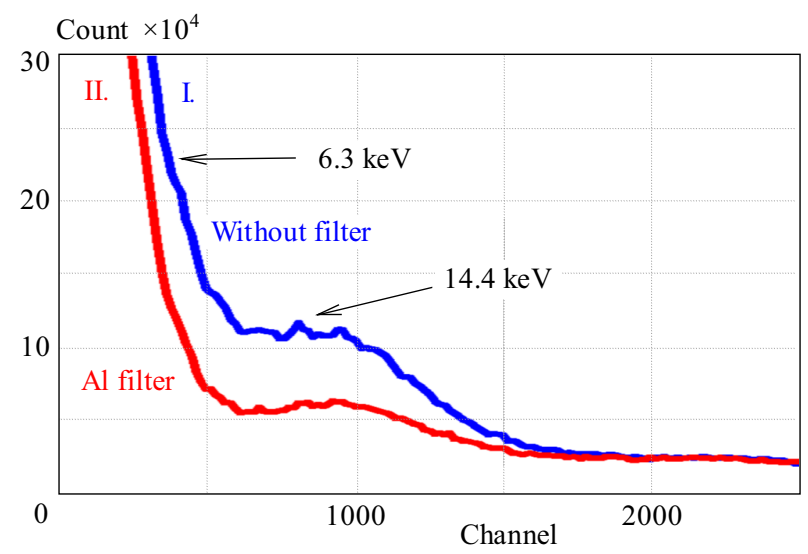

Fig. 9. YAP:Ce signal MCA acquired using IND internal SCA

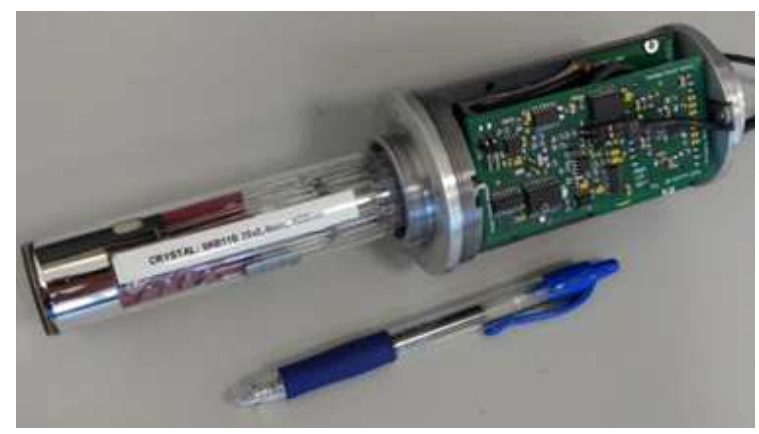

Fig. 10. The final look of the IND system (without housing), compared to a pen

As it is shown in Tab. 1, Mössbauer effect is the lowest for the lowest and the highest time delays. The delay increasing causes, the effect increasing up to its maximum. Further increase of the delay leads to the effect decreasing. The same applies for the Mössbauer spectrum quality parameter. It means that the best performance of this detection system has been proven to be a compromise between width and distinguishability of the $14.4 \mathrm{keV}$ photopeak and so $55 \mathrm{~ns}$ was selected as the best parameter.

In Fig. 8, the Mössbauer spectrum of the natural iron reference absorber registrated using optimized IND detection system is presented.

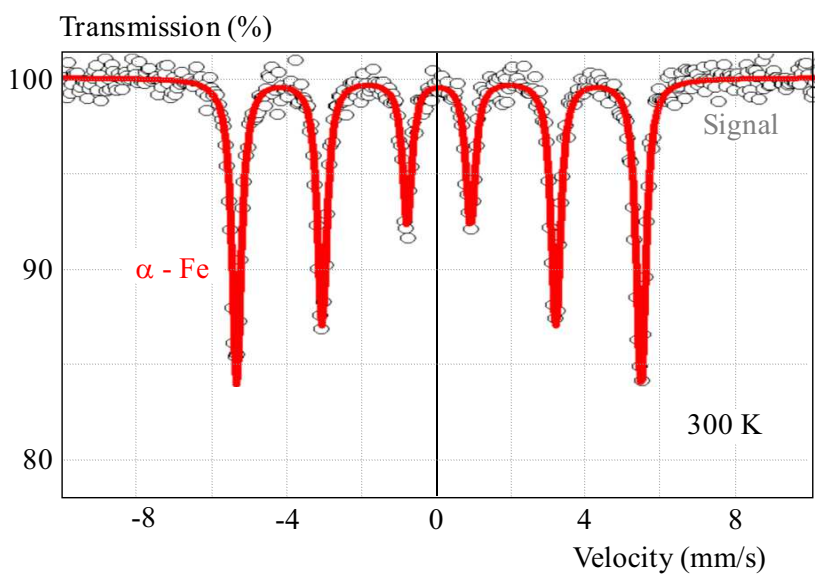

Fig. 8. Mössbauer spectrum of the natural iron reference absorber

\subsection{Final IND internal SCA performance test}

After complete IND detection system optimization, its MCA was recorded (see Fig. 9).

The performance of the IND internal SCA was compared also with the MS'96 SCA and the corresponding Mössbauer spectra of the natural iron reference absorber were measured. The results of this comparison are shown in Tab. 2. It is obvious that the usage of the IND internal SCA provides a significantly higher spectrum quality (improvement by $15 \%$ ) and Mössbauer effect (improvement by $7 \%)$.

\section{CONCLUSION AND DISCUSSION}

The precise compact system for ionizing radiation detection and signal processing with advanced components integration and electronic control has been developed [20] and successfully implemented into current experimental setups of Mössbauer spectrometers. The IND system is compact and effective. It contains the scintillation detector (YAP:Ce crystal), the high voltage supply, the preamplifier, the amplifier, the single-channel analyzer, the counter and the microcontrollers with memory. Thanks to the electronic control, self-backup of operating parameters and internal temperature self-monitoring it is called intelligent. The IND system has both analog and logic output.

The IND detection system has been compared to the National Instruments digitizer and to the original MS'96 Mössbauer spectrometer and exhibits valuable improvement. From now, Mössbauer spectrometers in the RCPTM laboratories are equipped with the IND detection system. Complete IND detection system is shown in Fig. 10.

\section{Acknowledgement}

The authors acknowledge support from the Ministry of Education, Youth and Sports of the Czech Republic (LO1305). Authors thank to internal IGA grant of Palacky University $\left(\mathrm{IGA}_{P} r F_{2} 015_{0} 17\right)$. 


\section{REFERENCES}

[1] YI, Q.-HONG, S.-JIE, K.-CHENG-FU, D.-XIAO-LI, M. -XIAO-GANG, L.: Development of Front-End Readout Electronics for Silicon Strip, Chinese Physics C 37 No. 1 (2013), 016101.

[2] ESPOSITO, B.-RIVA, M.-MAROCCO, D.-KASCHUCK, Y.: A Digital Acquisition and Elaboration System for Nuclear Fast Pulse Detection, Nuclear Instruments and Methods A No. 572 (2007), 355 .

[3] ENGEL, G. L.-SADASIVAM, M.-NETHI, M.-ELSON, J. M.-SOBOTKA, L. G.-CHARITY, R. J.: A Multi-Channel Integrated Circuit for Use in Low-And Intermediate-Energy Nuclear Physics - HINP16C, Nuclear Instruments and Methods A No. 573 (2007), 418.

[4] PROCHAZKA, R.-MASLAN, M.-PECHOUSEK, J.-JANCIK, D.: Optimizing the Features of the Single-Channel Analyzer towards Improvement of Productivity of Mössbauer Spectra, Czechoslovak Journal of Physics No. 56 (2006), E133.

[5] PROCHAZKA, R.-PECHOUSEK, J.-FRYDRYCH, J. : Development of the Fast Scintillation Detector with Programmable High Voltage Adjustment Suitable for Möossbauer Spectroscopy, AIP Conference proceedings, 2010, p. 143.

[6] KWANG, H. K.-IN, S. J.-HO-SIK, Y. : Portable Gamma-Ray Tracking System for Nuclear Material Safety, Nuclear Instruments and Methods A No. 607 (2007), 64.

[7] Patent CN 102798881 (A), Nuclear radiation detection system, Ningbo Chi Ming Core Electronic Technology Co., Ltd.: Li Z., Application number CN 201210298203.

[8] Patent FR 2973116 (A1), Nuclear radiation detection device for use in key chain for detection of gamma and beta particles during disaster at nuclear power plant, Gregory J., Application number FR20110000853 20110322.

[9] Patent US 2004251422 (A1), Ionization chamber detector system for the detection and measurement of nuclear radiation including beta, gamma, and x-ray radiation, Overhoff M., Application number US 10/766,717.

[10] Patent US 2007145260 (A1), Instrumentation package and integrated radiation detector, General Electric Company: Frederick L., Medley D., Glesius F., Application number US 11/319,581.

[11] Patent WO 9807110 (A1), Ambient temperature cadmium zinc telluride radiation detector and amplifier circuit, Univ. California: Lavietes A. D., Mcquaid J. H., Application number $\mathrm{PCT} / \mathrm{US1997/013452.}$

[12] Patent US 4336532 (A), Integrated nuclear radiation detector and monitor, Radiation Engineering Inc.: Biehl B. L., Lieberman S. I., Application number US 06/152,273.

[13] MASHLAN, M.-JANCIK, D.-KHOLMETSKII, A.-MULABA, A.-POLLAK, H.: Mössbauer Spectrometer with YAlO3: Ce Scintillation Crystal, Hyperfine Interactions 120/121, 769.

[14] Patent CZ 302439 B6, Mössbauer Spectrometer, Palacky University Olomouc: Zboril R., Mashlan M., Kholmetskii A., Yevdokimov V., Application number CZ20050000422 20050629.

[15] Patent CZ 302515 B6, Mover for Mössbauer Spectrometer with Resonance Detection of Gamma Radiation, Palacky University Olomouc: Mashlan M., Pechousek J., Yevdokimov V., Application number CZ20040001064 20041022.
[16] Patent CZ 302779 B6, Mössbauer Spectrometer, Palacky University Olomouc: Prochazka R., Mashlan M., Tucek J., Tucek P., Pechousek J., Application number CZ20100000380 20100517.

17] PECHOUSEK, J.-NOVAK, P.-NAVARIK, J.-KOHOUT, P.-MACHALA, L. : Mössbauer Spectroscopy System with Increased Performance and Flexibility - Utilization in Material Research, Journal of Electrical Engineering 64 No. 6 (2013), 386.

18] PECHOUSEK, J.-PROCHAZKA, R.-PROCHAZKA, V.FRYDRYCH, J.: Virtual Instrumentation Technique used in the Nuclear Digital Signal Processing System Design: Energy and Time Measurement Tests, Nuclear Instruments and Methods A No. 637 (2011), 200.

[19] NOVAK, P.-NAVARIK, J.-PECHOUSEK, J.-PROCHAZKA, V.-MACHALA, L.-TUCEK, J. : Development of Fast Pulse Processing Algorithm for Nuclear Detectors and its Utilization in LabVIEW-Based Mössbauer spectrometer, Journal of Instrumentation 9 (2014), article number T01001.

[20] Patent CZ 25467 U1, System for Detection of Ionizing Radiation, Palacky University Olomouc: Navarik J., Novak P., Pechousek J., Jancik D., Machala L., Mashlan M., Application number CZ20130028339U 20130820.

Received 12 November 2014

Jakub Navařík is PhD student of Faculty of Science of the Palacký University in Olomouc. He also received his Master degree in applied physics there. He is researcher at Regional Centre of Advanced Technologies and Materials. His scientific focus is Mössbauer spectroscopy, its instrumentation and electronics.

Petr Novák is PhD student of Faculty of Science of the Palacký University in Olomouc. He also received his Master degree in applied physics there. He is researcher at Department of Experimental Physics. His scientific interests are Mössbauer spectroscopy and virtual instrumentation.

Jiří Pechoušek is associate professor of Faculty of Science of the Palacký University in Olomouc. He also received his $\mathrm{PhD}$ degree in applied physics there. He is researcher at both Department of Experimental Physic and Regional Centre of Advanced Technologies and Materials. His scientific focus is Mössbauer spectroscopy and virtual instrumentation.

Libor Machala is associate professor of Faculty of Science of the Palacký University in Olomouc. He also received his $\mathrm{PhD}$ degree in applied physics there. He is researcher of both Department of Experimental Physic and Regional Centre of Advanced Technologies and Materials. His scientific focus is Mössbauer spectroscopy and material physics.

Dalibor Jančík is researcher at Regional Centre of Advanced Technologies and Materials. His scientific focus is electronics and Mössbauer spectroscopy.

Miroslav Mašlán̆ is professor of Faculty of Science of the Palacký University in Olomouc. He is researcher at Department of Experimantal Physic. His scientific focus is Mössbauer spectroscopy. 